\title{
Plasma Drug Concentrations in Patients with Pulmonary Arterial Hypertension on Combination Treatment
}

\author{
Ekkehard Grünig $^{a} \quad$ Johanna Ohnesorge ${ }^{a} \quad$ Nicola Benjamin $^{a} \quad$ Jürgen Burhenne \\ Yeliz Enderle $^{c}$ Benjamin Egenlauf ${ }^{a}$ Christine Fischer $^{b}$ Satenik Harutyunova ${ }^{a}$ \\ Andrea Huppertz ${ }^{c}$ Hans Klose ${ }^{d}$ Walter E. Haefelic \\ ${ }^{a}$ Center for Pulmonary Hypertension, Thorax Clinic at the University Hospital Heidelberg, ${ }^{b}$ Institute of Human \\ Genetics, University of Heidelberg, and ' Department of Clinical Pharmacology and Pharmacoepidemiology, \\ University Hospital Heidelberg, Heidelberg, and d Section of Pneumology, University Hospital Hamburg, \\ Hamburg, Germany
}

\section{Keywords}

Pulmonary arterial hypertension · Combination therapy · Sildenafil · Tadalafil · Bosentan · Ambrisentan · Macitentan

\begin{abstract}
Background: Combination therapy with the phosphodiesterase type 5 inhibitors (PDE-5i) sildenafil or tadalafil and the endothelin receptor antagonists (ERA) bosentan, ambrisentan, or macitentan may cause mutual pharmacokinetic interactions in patients with pulmonary arterial hypertension (PAH). Objective: The objective of this study was to analyze plasma drug concentrations in $\mathrm{PAH}$ patients receiving different combination treatments. Methods: $\mathrm{PAH}$ patients receiving a stable combination treatment with ERA and PDE-5i with targeted dosage for at least 1 month were routinely assessed, including clinical parameters and plasma drug concentrations. Concentrations were normalized considering dose and time from last medication intake and presented as multiples of the expected mean (MoM) of the respective monotherapies. Results: A total of $125 \mathrm{PAH}$ patients (84 female, 41 male, 57\% idiopathic/heritable) were included. Sildenafil and tadalafil concentrations were lowest in combina-
\end{abstract}

\section{KARGER}

(C) 2017 S. Karger AG, Basel

E-Mail karger@karger.com

www.karger.com/res tion with bosentan (MoM $0.44 \pm 0.42,95 \%$ confidence interval [Cl] 0.30-0.57, and MoM $0.89 \pm 0.53,95 \% \mathrm{Cl} 0.50-1.28$, respectively) compared to the combination with ambrisen$\tan$ (MoM 1.3 $\pm 0.97,95 \%$ Cl 0.86-1.73, and MoM 1.67 \pm 0.63 , $95 \% \mathrm{Cl} 1.40-1.94$, respectively) and macitentan (MoM $1.16 \pm$ $0.87,95 \% \mathrm{Cl} 0.86-1.46$, and MoM $1.59 \pm 0.99,95 \% \mathrm{Cl} 0.80-$ 2.38 , respectively). The combination of sildenafil and bosentan led to more than twice the expected bosentan concentrations in 53.8\%. Patients switching from sildenafil-bosentan to macitentan showed a significant increase in sildenafil concentrations ( $p<0.001)$. Conclusions: Only the combination with macitentan or ambrisentan led to targeted mean PDE-5i plasma concentrations and should therefore be preferred to combination with bosentan. Sildenafil-bosentan showed the strongest interaction, with low sildenafil and high bosentan concentrations. The study was not powered to analyze whether lower PDE-5i concentrations cause unsatisfying clinical response. However, plasma concentrations within a targeted range are desirable and may become of increasing importance.

(c) 2017 S. Karger AG, Basel

E. Grünig and J. Ohnesorge contributed equally to this work.

Prof. Dr. med. Ekkehard Grünig

Center for Pulmonary Hypertension

Thorax Clinic at the University Hospital Heidelberg

Röntgenstr. 1, DE-69126 Heidelberg (Germany)

E-Mail ekkehard.gruenig@ med.uni-heidelberg.de 


\section{Introduction}

Within the last 15 years, 11 targeted drugs for the medical treatment of pulmonary arterial hypertension $(\mathrm{PAH})$ have been approved in many countries [1], and the possibility to combine 2 or more classes of drugs has become increasingly relevant. Three separate signaling pathways of the disease can be addressed by specific drugs such as prostanoids, soluble guanylate cyclase stimulators, endothelin receptor antagonists (ERA; bosentan, macitentan, and ambrisentan), and phosphodiesterase type 5 inhibitors (PDE-5i; sildenafil and tadalafil). With increasing use of combination therapy, drug-drug interactions have to be considered.

In the recently published COMPASS- 2 trial, combination therapy with bosentan and sildenafil compared with sildenafil and placebo did not show an improvement of the composite primary endpoint time to clinical worsening [2]. Insufficient sildenafil drug plasma concentrations due to pharmacokinetic interactions between bosentan and sildenafil were discussed as a possible reason for the lack of efficacy, but were not tested [2]. Similar results were obtained for the combination of tadalafil and bosen$\tan$ in the PHIRST study, with a less pronounced effect in patients pretreated with bosentan compared to treatment-naïve patients, which may possibly be attributed to a decrease in tadalafil plasma concentrations when coadministered with bosentan [3].

The combination of bosentan and sildenafil is one of the most common combination treatments for PAH in adults worldwide [4]. Drug-drug interactions of this medication combination resulting in different plasma concentrations would be of significant clinical impact. In healthy subjects $[5,6]$ and in PAH patients $[7,8]$, it has already been shown that bosentan significantly reduces the maximum plasma concentration of sildenafil by approximately $50 \%$ and that of tadalafil by up to $26 \%$. Conversely, sildenafil inhibits hepatic uptake of bosentan by inhibition of organic aniontransporting polypeptides [9]. Therefore, bosentan plasma concentration increased by $\sim 40 \%$ when bosentan was coadministered with sildenafil in healthy subjects [5], but was not affected when combined with the weaker organic anion-transporting polypeptide inhibitor tadalafil $[6,10]$. Due to this known interaction, the package inserts of both bosentan and sildenafil inform that the combination of the 2 agents leads to an increase in sildenafil and a decrease in bosentan, but is not thought to require dose adjustments. In healthy subjects, it has been shown that the interaction between sildenafil and bosentan becomes stronger over time, with a more pronounced decrease in sildenafil and an

Plasma Drug Concentrations in Patients with Pulmonary Arterial Hypertension increase in bosentan concentrations after 16 days, compared to 6 or 10 days of combination treatment [5]. This might be due to the time of the bosentan-mediated enzyme induction of cytochrome P450 (CYP) isozymes and the synthesis of new enzymes, which are responsible for the degradation of most lipophilic drugs such as sildenafil and bosentan [11-13]. However, medication interactions of combination treatment for at least 1 month have not yet been investigated in a larger cohort of PAH patients. In contrast, in dedicated studies in healthy volunteers, an interaction of ambrisentan with sildenafil or tadalafil was ruled out $[14,15]$, whereas the coadministration of macitentan with sildenafil mildly increased sildenafil peak concentrations by $26 \%$ [16].

Most pharmacokinetic interaction studies are conducted in healthy volunteers and typically assess a shortterm combination therapy early after or even before reaching steady-state conditions. Therefore, the importance of pharmacokinetic interactions of the long-term combination of PDE-5i and ERA in clinical practice remains unclear. In other areas, the observed extent of interaction was smaller than predicted from studies in healthy volunteers (e.g., direct oral anticoagulants [17]).

The objective of this study was to analyze plasma drug concentrations in $\mathrm{PAH}$ patients receiving various combination treatments with a stable dose of different ERA and PDE-5i for at least 1 month. Furthermore, we aimed to assess changes in ERA and PDE-5i plasma concentrations after switching to a different ERA due to clinical worsening or lack of clinical improvement. We also analyzed the potential impact of comorbidity (connective tissue disease [CTD], renal impairment with estimated glomerular filtration rate $<60 \mathrm{~mL} / \mathrm{min} / 1.73 \mathrm{~m}^{2}$ ) and coadministration of imatinib on PAH drug exposure.

Imatinib is an antiproliferative agent that was developed for the treatment of chronic myeloid leukemia. As this agent also shows inhibitory effects on the plateletderived growth factor and c-KIT signaling, it was suggested that it may be efficacious in $\mathrm{PAH}$, but it was not approved due to side effects and serious adverse events [18]. Patients who participated in the phase III trial often continued treatment with imatinib due to good clinical response and positive effects on quality of life [19].

\section{Subjects and Methods}

Study Population and Design

Patients with invasively confirmed PAH who received targeted combination treatment were consecutively included from March 2015 on by clinical routine examinations and assessment of medi- 
cation plasma concentrations in the Center for Pulmonary Hypertension, Thorax Clinic at the University Hospital Heidelberg. Patients with former routine assessments of medication plasma concentrations were asked for their permission to retrospectively include these results and corresponding clinical data. PAH-targeted treatment had to be on a stable dose for at least 1 month before plasma concentration measurement. Patients entered the study on their individually established dose of PAH medication, and neither patients nor clinicians knew the drug plasma concentrations. All decisions about drug dosing were uninfluenced by the concentration measurements.

Clinical routine assessments comprised medical history, concomitant medication, concomitant diseases, demography, vital signs, electrocardiography, echocardiography, routine blood tests including NT-proBNP, and 6-min walking distance as described previously $[20,21]$. The data of the most recent right heart catheterization were used for hemodynamics.

In remnants of routine blood samples that were taken before the next scheduled combination therapy intake, the plasma concentrations of sildenafil, tadalafil, bosentan, and ambrisentan were measured using validated, sensitive mass spectrometry (LC-MS/ MS) methods as previously described [22]. At the time of analysis, the measurement of macitentan concentrations was not methodologically established. Dosing schedule and last intake were documented for later interpretation of the results. Potentially interacting comedication with targeted $\mathrm{PAH}$ drugs was checked using the AiDKlinik software [23].

Elimination organ dysfunctions such as hepatic and renal impairment were diagnosed by a specialist physician (nephrologist, gastroenterologist) and presented among other criteria of respective organ dysfunction with creatinine $>1.1 \mathrm{mg} / \mathrm{dL}$ or glomerular filtration rate $<60 \mathrm{~mL} / \mathrm{min} / 1.73 \mathrm{~m}^{2}$ (renal impairment), or increased gamma-glutamyl transferase $\geq 60 \mathrm{U} / \mathrm{L}$ for male patients and $>40 \mathrm{U} / \mathrm{L}$ for female patients (hepatic impairment). The study had a positive unrestricted vote of the responsible Ethics Committee of the Medical Faculty of Heidelberg University (ID S-605/2014), and all patients gave their written informed consent for participation in the study. The study was registered at clinicaltrials.gov (NCT02484807).

Normalization of Measurements: Multiple of the Expected Mean Individual plasma concentrations were interpreted in relation to the expected mean medication concentrations at the respective sampling time point in the dosing interval. The expected mean concentration ranges refer to data extracted from published plasma concentration-time profiles measured during monotherapy with sildenafil [24, 25], tadalafil [26], bosentan [27-29], and ambrisentan [30] and served as comparative values. To calculate doseand time-specific expected mean values, dose proportionality was assumed for each agent according to the literature [26, 31-33]. Each individually measured drug concentration was set in proportion to the expected mean concentration and expressed as a multiple of the expected mean (MoM), with values $<1$ denoting lower and values $>1$ higher values than the expected mean. For better orientation and interpretation, target ranges of plasma drug concentrations were defined. The commonly used ranges to prove bioequivalence of generic drugs are $80-125 \%$ regarding the maximum concentration or area under the curve of medication concentrations. The bioequivalence ranges derive from the FDA Guidance (Guidance for Industry. Drug Interaction Studies - Study Design, Data Analysis, Implications for Dosing, and Labeling Recommen- dations) as an initial cutoff for an inhibitor/inductor. To define ranges with marked difference from expected values, we introduced a conservative definition for the targeted medication concentrations representing a more distinct inhibition/induction at one point in time. The ranges were expanded to 0.5-2.0 times the expected mean plasma concentration, corresponding to half (lower limit $=0.5$ ) or twice (upper limit $=2.0$ ) the expected mean plasma concentrations in order to achieve a conservative assumption of targeted medication concentrations.

\section{Statistical Analysis}

The analyses were performed by 2 statisticians (N. Benjamin, C. Fischer). Data are presented as mean \pm standard deviation or sample size and respective percentage. Additionally, 95\% confidence intervals (CIs) are given for effect size estimation. Due to the exploratory nature of this study, no sample size analysis was conducted in advance of the trial.

Differences in MoM between groups were analyzed by ANOVA. Individual comparisons between single combination treatment groups and analysis of influencing factors were performed by the Wilcoxon rank-sum test. Plasma concentrations before and after transition were compared by the Wilcoxon signed-rank test.

A sensitivity analysis excluding patients with known influencing factors such as concomitant disease or known interacting comedications was performed to rule out the effect of known interactions. Furthermore, a potential influence of individual drug doses on drug concentrations was analyzed by ANOVA with the drug combination as fixed factor. The association of clinical data and medication concentrations was analyzed by Pearson correlation analysis using clinical data from 6-min walking distance, echocardiographic parameters, and blood gas analysis.

All tests were 2 -sided and $p$ values $<0.05$ were considered statistically significant. All analyses were carried out with IBM SPSS V22 (IBM Corp., Armonk, NY, USA).

\section{Results}

The clinical characteristics of the study cohort are given in Table 1. Plasma drug concentrations were assessed in 125 patients; $67 \%$ were female, $57 \%$ had idiopathic/ heritable $\mathrm{PAH}, 10.4 \%$ had congenital heart disease-associated PAH, 21\% had CTD-associated PAH, and 11.6\% had PAH with comorbidities (pre- and postcapillary components of pulmonary hypertension due to additional comorbidity, atypical PAH). Mean pulmonary arterial pressure was $47 \pm 15 \mathrm{~mm} \mathrm{Hg}$, and $43.4 \%$ were in WHO functional class (WHO-FC) II, 53.3\% in WHO-FC III, and $3.3 \%$ in WHO-FC IV. They were receiving targeted PAH combination treatment for a median of 14 months (ERA, range 1-194 months) and 13 months (PDE-5i, range 2-194 months). All plasma concentrations were measured at trough before the next planned medication intake. Renal insufficiency was reported in $44(35.2 \%)$ patients, hepatic insufficiency based on elevated gamma-
28

Respiration 2017;94:26-37

DOI: $10.1159 / 000470916$
Grünig et al. 
Table 1. Clinical characteristics of the study cohort at baseline at first ERA/PDE-5i measurement

\begin{tabular}{|c|c|c|c|c|c|c|}
\hline & $\begin{array}{l}\text { Bosentan- } \\
\text { sildenafil }\end{array}$ & $\begin{array}{l}\text { Ambrisentan- } \\
\text { sildenafil }\end{array}$ & $\begin{array}{l}\text { Macitentan- } \\
\text { sildenafil }\end{array}$ & $\begin{array}{l}\text { Bosentan- } \\
\text { tadalafil }\end{array}$ & $\begin{array}{l}\text { Ambrisentan- } \\
\text { tadalafil }\end{array}$ & $\begin{array}{l}\text { Macitentan- } \\
\text { tadalafil }\end{array}$ \\
\hline Total number & 39 & 19 & 33 & 7 & 21 & 6 \\
\hline \multicolumn{7}{|l|}{ Demography } \\
\hline Age, years & $56 \pm 16$ & $61 \pm 19$ & $65 \pm 13$ & $56 \pm 19$ & $63 \pm 13$ & $64 \pm 21$ \\
\hline Height, cm & $166 \pm 10$ & $165 \pm 9$ & $169 \pm 9$ & $166 \pm 11$ & $168 \pm 6$ & $172 \pm 7$ \\
\hline Weight, kg & $74 \pm 22$ & $75 \pm 17$ & $81 \pm 19$ & $73 \pm 16$ & $77 \pm 17$ & $72 \pm 9$ \\
\hline Female sex & $29(74.4 \%)$ & $15(78.9 \%)$ & $19(57.6 \%)$ & $5(71.4 \%)$ & $12(57.1 \%)$ & $4(66.7 \%)$ \\
\hline Body mass index & $27.0 \pm 7.5$ & $27.4 \pm 5.6$ & $28.1 \pm 5.8$ & $26.5 \pm 5.5$ & $27.1 \pm 4.5$ & $24.7 \pm 3.1$ \\
\hline \multicolumn{7}{|l|}{ Medication } \\
\hline Daily ERA dose, $\mathrm{mg} / \mathrm{kg}$ & $240 \pm 35$ & $8 \pm 3$ & $10 \pm 0$ & $250 \pm 0$ & $9 \pm 3$ & $10 \pm 0$ \\
\hline Daily PDE-5i dose, $\mathrm{mg} / \mathrm{kg}$ & $178 \pm 89$ & $147 \pm 90$ & $73 \pm 26$ & $43 \pm 8$ & $37 \pm 10$ & $27 \pm 10$ \\
\hline \multicolumn{7}{|l|}{ Diagnosis } \\
\hline IPAH & 20 & 9 & 17 & 3 & 12 & 1 \\
\hline НРAH & 5 & 2 & 1 & 1 & 0 & 0 \\
\hline CHD-associated PAH & 5 & 1 & 5 & 2 & 0 & 0 \\
\hline CTD-associated PAH & 4 & 5 & 4 & 0 & 9 & 4 \\
\hline Atypical PAH & 5 & 2 & 6 & 1 & 0 & 1 \\
\hline \multicolumn{7}{|l|}{ Hemodynamics } \\
\hline Mean PAP (RHC), mm Hg & $53 \pm 15$ & $49 \pm 16$ & $41 \pm 14$ & $44 \pm 14$ & $45 \pm 15$ & $36 \pm 11$ \\
\hline Cardiac output, $\mathrm{L} / \mathrm{min}$ & $4.9 \pm 1.5$ & $4.7 \pm 1.4$ & $4.8 \pm 1.7$ & $4.5 \pm 1.2$ & $4.8 \pm 1.9$ & $5.1 \pm 1.1$ \\
\hline PVR, dynes $\times \mathrm{s} \times \mathrm{cm}^{-5}$ & $693 \pm 287$ & $649 \pm 400$ & $503 \pm 317$ & $501 \pm 339$ & $669 \pm 401$ & $411 \pm 335$ \\
\hline Systolic PAP (Echo), mm Hg & $64 \pm 20$ & $59 \pm 23$ & $48 \pm 19$ & $60 \pm 29$ & $43 \pm 14$ & $40 \pm 15$ \\
\hline \multicolumn{7}{|l|}{ Exercise capacity } \\
\hline Six-minute walking distance, $\mathrm{m}$ & $399 \pm 120$ & $371 \pm 110$ & $392 \pm 115$ & $408 \pm 113$ & $397 \pm 97$ & $395 \pm 100$ \\
\hline \multicolumn{7}{|l|}{ Laboratory } \\
\hline NT-proBNP, ng/mL & $2,023 \pm 3,304$ & $2,756 \pm 6,813$ & $594 \pm 782$ & $1,287 \pm 1,247$ & $2,073 \pm 5,892$ & $5,019 \pm 9,516$ \\
\hline PDE-5i concentration, MoM & $0.44 \pm 0.42$ & $1.30 \pm 0.97$ & $1.16 \pm 0.87$ & $0.89 \pm 0.53$ & $1.67 \pm 0.63$ & $1.59 \pm 0.99$ \\
\hline ERA concentration, MoM & $3.54 \pm 3.24$ & $1.81 \pm 0.82$ & not assessable & $2.18 \pm 1.23$ & $1.53 \pm 0.84$ & not assessable \\
\hline
\end{tabular}

Values are presented as mean \pm standard deviation or $n(\%)$. CHD, congenital heart disease; CTD, connective tissue disease; Echo, echocardiography; ERA, endothelin receptor antagonist; HPAH, heritable pulmonary arterial hypertension; IPAH, idiopathic pulmonary arterial hypertension; MoM, multiple of the expected mean; NT-proBNP, N-terminal prohormone of brain natriuretic peptide; PAH, pulmonary arterial hypertension; PAP, pulmonary arterial pressure; PDE-5i, phosphodiesterase type 5 inhibitor; PVR, pulmonary vascular resistance; RHC, right heart catheterization.

glutamyl transferase in 4 (3.2\%). None of the patients had significantly elevated transaminase levels. Echocardiography, 6-min walking distance, and NT-proBNP levels were done on the day of the routine visit, while hemodynamic parameters measured by right heart catheterization were obtained from the most recent invasive hemodynamics assessment (Table 1).

\section{Medication Plasma Concentrations in Different \\ Combinations of ERA and PDE-5i}

Plasma concentrations of PDE-5i significantly differed between the different combination treatments with ERA
(ERA-sildenafil, $p<0.001$; ERA-tadalafil, $p=0.042$; Fig. 1). Sildenafil and tadalafil concentrations were lowest in combination with bosentan (MoM $0.44 \pm 0.42,95 \%$ CI 0.30-0.57, and MoM $0.89 \pm 0.53$, 95\% CI 0.50-1.28, respectively) compared to the combination with ambrisentan (MoM 1.3 \pm 0.97, 95\% CI 0.86-1.73, and MoM 1.67 $\pm 0.63,95 \%$ CI 1.40-1.94, respectively) and macitentan (MoM $1.16 \pm 0.87,95 \%$ CI 0.86-1.46, and MoM $1.59 \pm$ $0.99,95 \%$ CI $0.80-2.38$, respectively; Fig. 1 ).

Bosentan plasma concentrations compared to ambrisentan concentrations were significantly higher in combination with sildenafil, while ERA concentrations 
Fig. 1. Boxplots showing the distribution of phosphodiesterase type 5 inhibitor (PDE5i) concentrations expressed as MoM in different combination treatments. Plasma concentrations significantly differed between the different endothelin receptor antagonists (ERA) for both combination with sildenafil (ANOVA, $p<0.001$ ) and tadalafil (ANOVA, $p=0.042$ ). PDE-5i concentrations were lowest in combination with bosentan. Boxplots represent the median as horizontal line, the lower and upper quartile as box, $1.5 \times$ interquartile range as whiskers, and outliers as dots and asterisks.

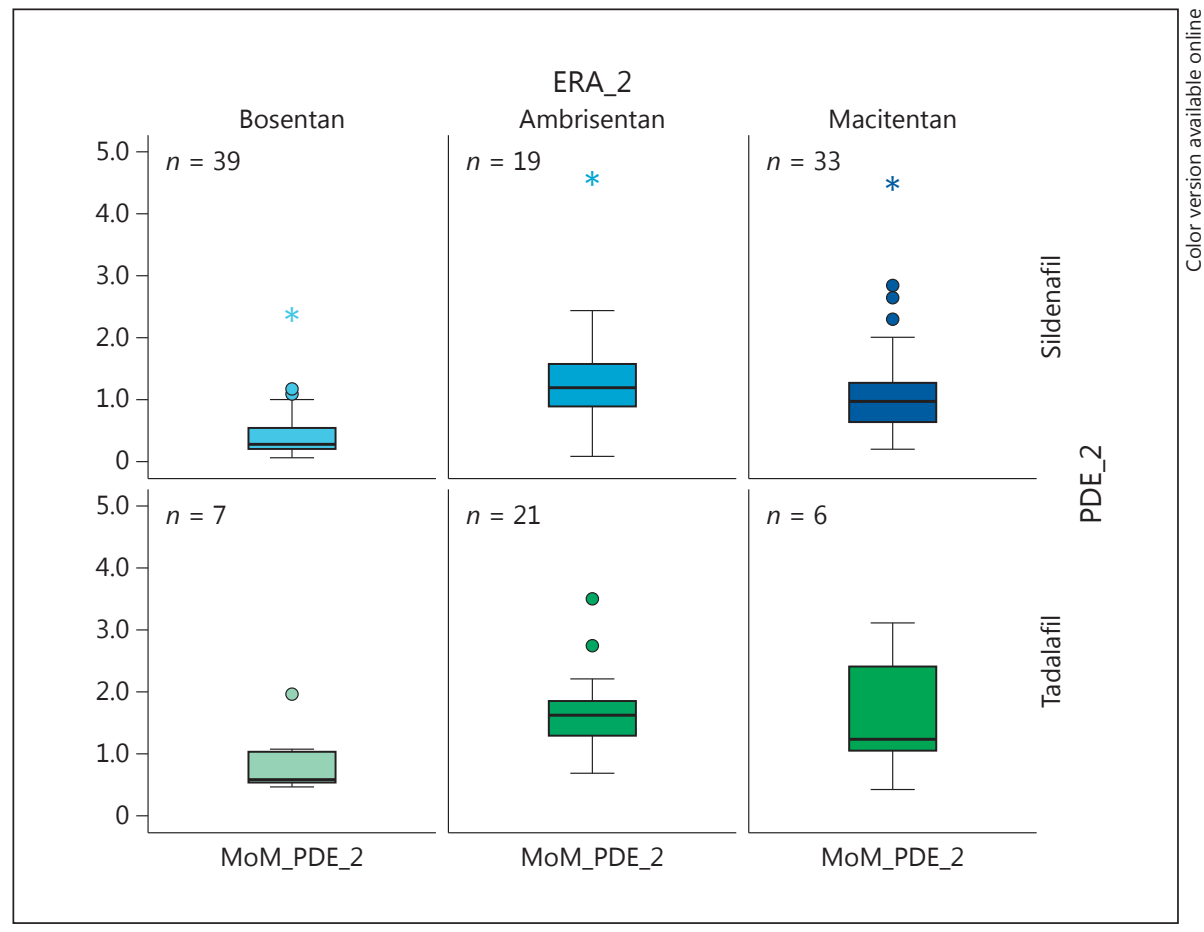

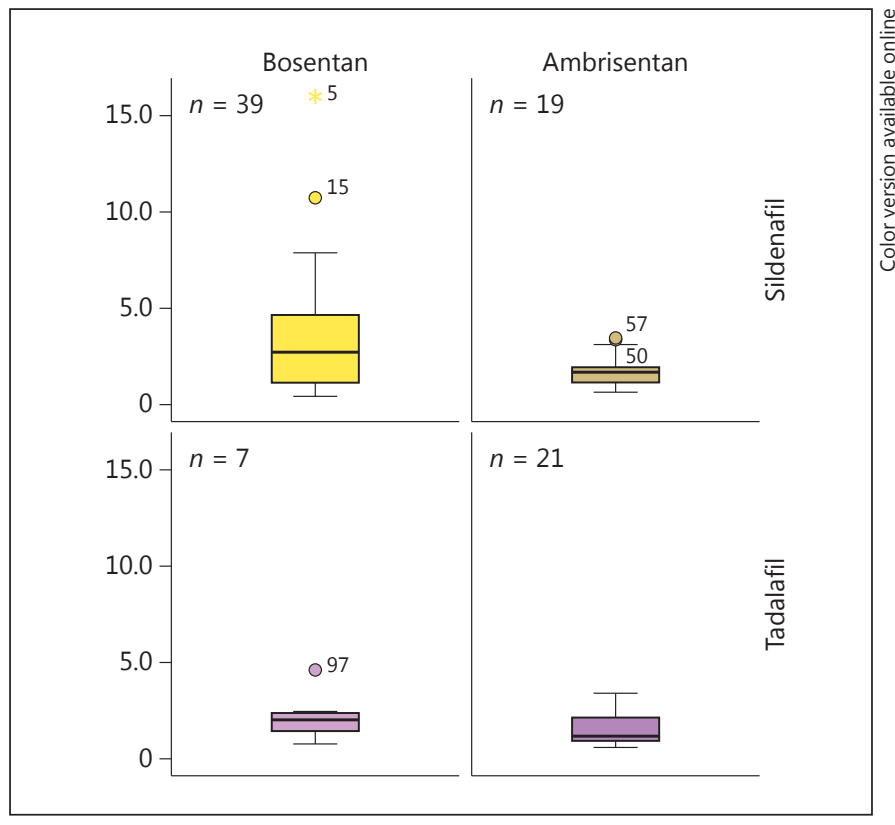

Fig. 2. Boxplots showing the distribution of endothelin receptor antagonist (ERA) concentrations expressed as MoM in different combination treatments with phosphodiesterase type 5 inhibitor. ERA concentrations significantly differed for sildenafil (ANOVA, $p=0.026$ ), but not for tadalafil (ANOVA, $p=0.129$ ). Boxplots represent the median as horizontal line, the lower and upper quartile as box, $1.5 \times$ interquartile range as whiskers, and outliers as dots and asterisks. did not differ when combined with tadalafil (ERA-sildenafil, ANOVA $p=0.026$; ERA-tadalafil, ANOVA $p=$ 0.129; Fig. 2).

Ranges of Drug Concentrations Using Multiples of the Expected Mean

The frequencies of plasma concentration ranges of PDE-5i for each combination treatment are given in Table 2 and for sildenafil-bosentan in Figure 3. In 28 out of 39 patients $(71.8 \%, 95 \%$ CI $57.7-85.9 \%)$ receiving combination treatment with sildenafil-bosentan, sildenafil concentrations were lower than half of the expected value, corresponding to a $<0.5 \mathrm{MoM}$, while individual concentrations for bosentan showed a $>2.0 \mathrm{MoM}$ in $53.8 \%$ of cases ( $n=21 / 39$, 95\% CI 38.2-69.5\%; Fig. 3). Thus, most of the patients receiving long-term combination treatment of sildenafil and bosentan (sildenafil $30 \pm 38$ months; bosentan $51 \pm 43$ months) presented with PDE-5i concentrations which were very low $(<0.5$ MoM; Fig. 3). In many of these patients only trace amounts of sildenafil were detected in plasma. In combination with other ERA, sildenafil concentrations were mostly in the range between 0.5 and $2.0 \mathrm{MoM}$ (ambrisentan, $n=14 / 19,73.7 \%$, 95\% CI 53.9-93.4\%; maciten$\tan , n=22 / 33,66.7 \%, 95 \%$ CI $50.6-82.8 \%)$. In these combinations, targeted sildenafil plasma concentra- 


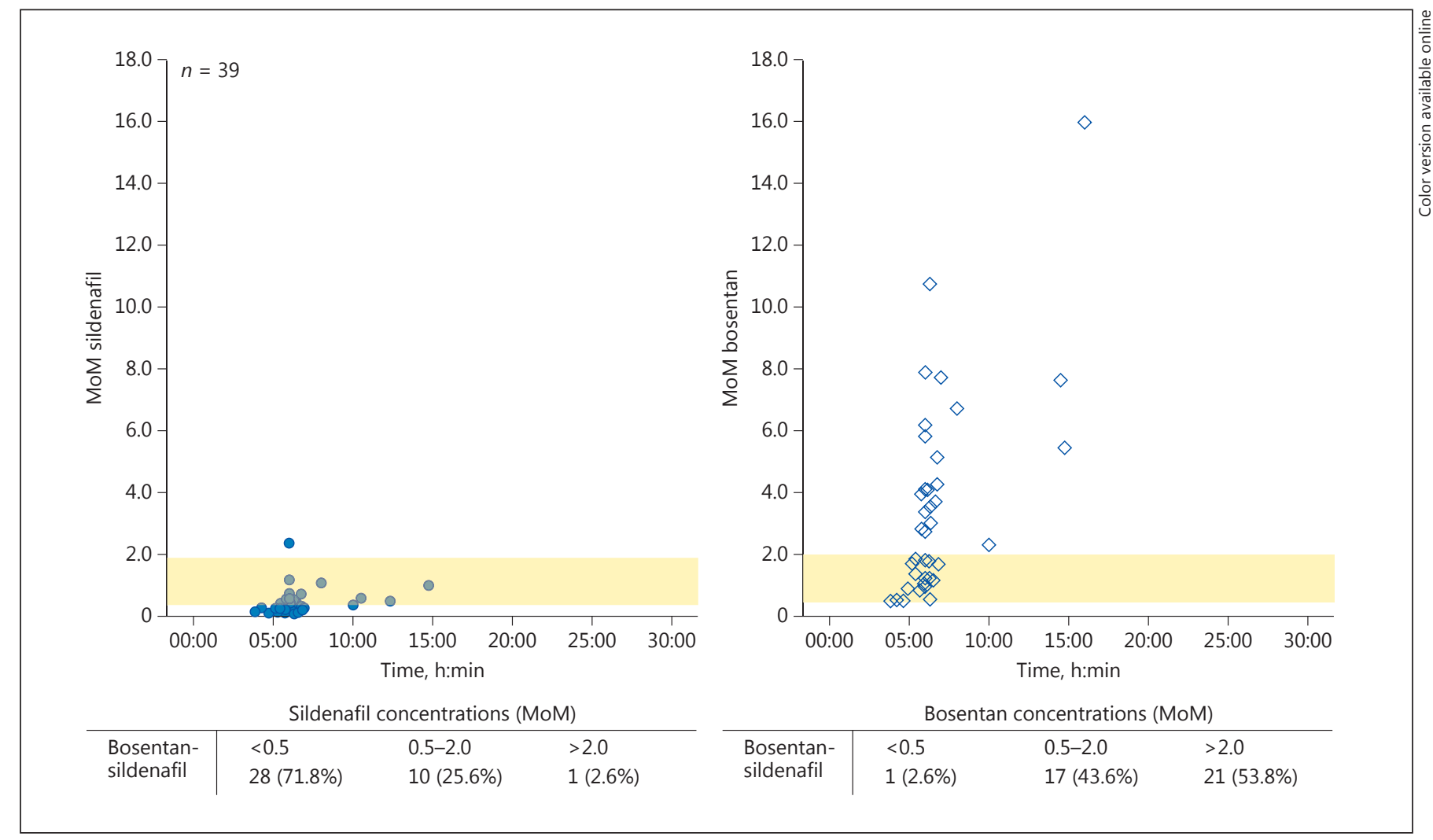

Fig. 3. In patients receiving sildenafil-bosentan, sildenafil concentrations were below half of the expected value in $71.8 \%$ of cases, whereas bosentan concentrations showed values above double the expected value in $>50 \%$ of cases. The MoM range between 0.5 (half of the expected value) and 2.0 (twice the expected value) is indicated as a shaded area.

tions were measured in almost all patients (Table 2; Fig. 4). However, even in the combination with other ERA, a few patients had levels $<0.5 \mathrm{MoM}$ (sildenafilambrisentan, $n=3 / 19$, 15.8\%; macitentan-sildenafil, $n=6 / 33,18.2 \%$; Table 2).

MoM of tadalafil were mainly in the target range of 0.5-2.0 MoM for combination with bosentan $(n=6 / 7$, $85.7 \%$, 95\% CI 59.8-100\%), except for 1 patient with MoM <0.5, ambrisentan ( $n=17 / 21,81.0 \%$, 95\% CI 64.297.7\%), and macitentan ( $n=3 / 5,50.0 \%, 95 \%$ CI $10-90 \%$; Table 2; Fig. 4).

\section{Factors Influencing Drug Concentrations}

The analysis of factors potentially influencing drug exposure included sex, elimination organ dysfunction, CTD, and comedication such as imatinib, treprostinil, or dipyrone. Furthermore, the relationship between ERA and PDE-5i dose/kg body weight and drug exposure was analyzed.

Plasma Drug Concentrations in Patients with Pulmonary Arterial Hypertension
Sex and renal and hepatic impairment did not affect plasma concentrations of ERA or PDE-5i in our study cohort. Patients with CTD $(n=26)$ had significantly lower drug concentrations than patients without CTD within each combination subgroup (ANOVA with medication combination as fixed factor, $p=0.04$; data not shown). Twenty-three patients (18.4\%) were on potentially interacting comedication; 5 patients $(4.0 \%)$ received imatinib, 17 (13.6\%) treprostinil (6 subcutaneously, 11 intravenously), and 1 (0.8\%) dipyrone. Imatinib was associated with significantly higher ERA plasma concentrations (MoM $4.72 \pm 1.73$, 95\% CI 3.05-6.44, vs. MoM $2.48 \pm$ 2.45, 95\% CI 1.95-3.01; ANOVA, $p=0.013)$. Treprostinil did not show a significant influence on medication plasma concentrations (ANOVA, $p=0.428$ ). Due to the small sample size, analysis for dipyrone with only 1 patient receiving sildenafil-ambrisentan was not performed.

Sildenafil doses/kg body weight varied depending on the combination partner. Significantly lower dosages $/ \mathrm{kg}$ 
Fig. 4. Plot showing the medication concentrations for phosphodiesterase type 5 inhibitors (PDE-5i) with respect to the applied dosages $/ \mathrm{kg}$ body weight. Significantly higher sildenafil dosages/kg body weight were given in patients receiving bosentan. The dosage/ $\mathrm{kg}$ body weight did not significantly influence the MoM plasma concentrations, with comparable MoM of sildenafil in the combination of sildenafilbosentan irrespective of the dosage/ $\mathrm{kg}$ and different MoM for comparable dosages $/ \mathrm{kg}$ for combination with other endothelin receptor antagonists (ERA).

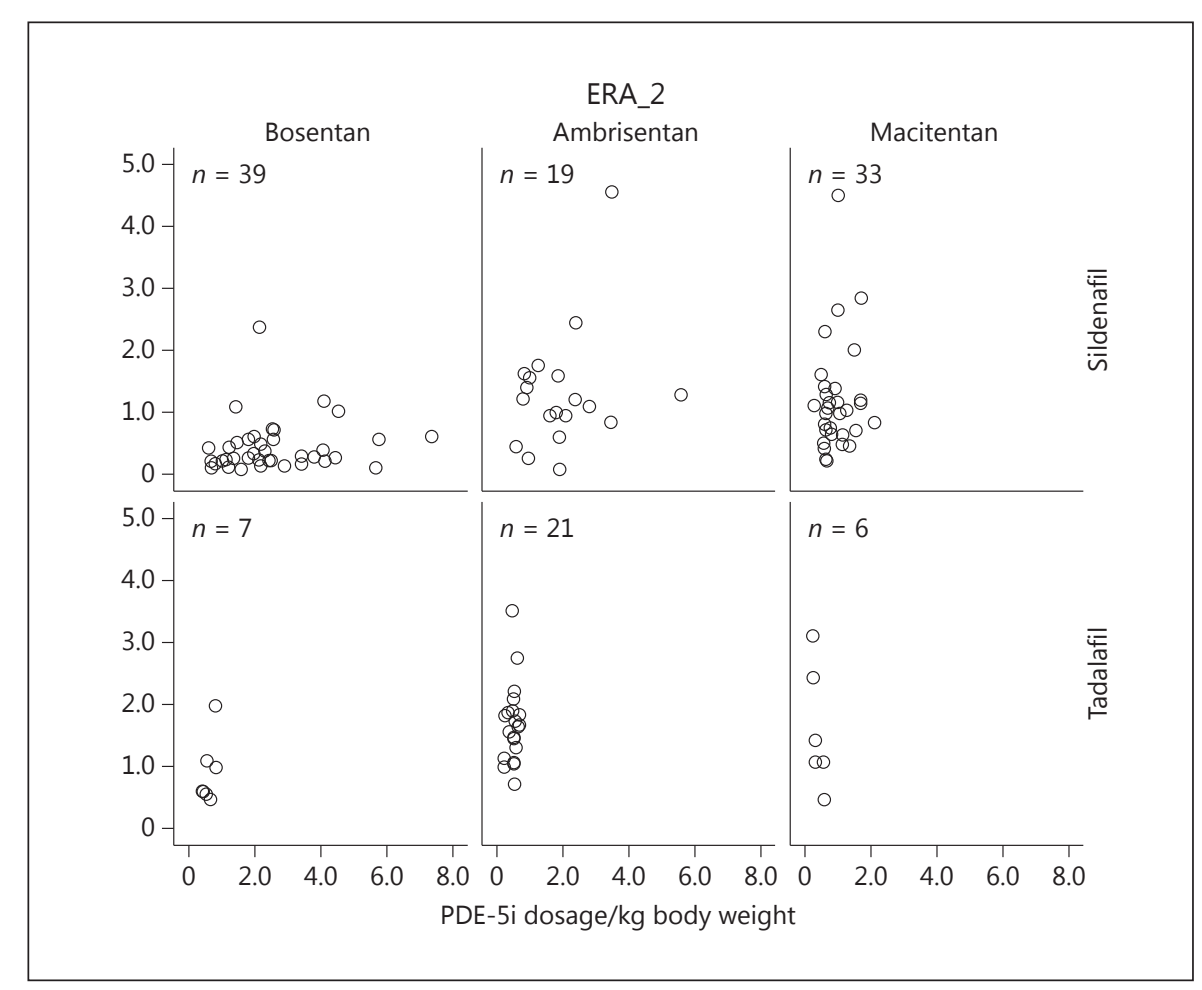

Table 2. Frequencies of PDE-5i plasma concentration ranges in different combination treatments

\begin{tabular}{lrrrrrr}
\hline & \multicolumn{2}{l}{ PDE-5i concentration, MoM } & & \\
\cline { 2 - 7 } & $<0.5$ & & $0.5-2$ & 2 \\
\hline Bosentan-sildenafil & 28 & $71.8 \%(57.7-85.9 \%)$ & 10 & $25.6 \%(11.9-39.4 \%)$ & 1 & $2.6 \%(0-7.5 \%)$ \\
Ambrisentan-sildenafil & 3 & $15.8 \%(0-32.1 \%)$ & 14 & $73.7 \%(53.9-93.5 \%)$ & 2 & $10.5 \%(0-24.3 \%)$ \\
Macitentan-sildenafil & 6 & $18.2 \%(5.0-31.3 \%)$ & 22 & $66.7 \%(50.6-82.8 \%)$ & 5 & $15.2 \%(2.9-27.4 \%)$ \\
\hline Bosentan-tadalafil & 1 & $14.3 \%(0-40.2 \%)$ & 6 & $85.7 \%(59.8-100 \%)$ & 0 & $0 \%$ \\
Ambrisentan-tadalafil & 0 & $0 \%$ & 17 & $81.0 \%(64.2-97.7 \%)$ & 4 & $19.0 \%(2.3-35.8 \%)$ \\
Macitentan-tadalafil & 1 & $16.7 \%(0-46.5 \%)$ & 3 & $50.0 \%(10.0-90.0 \%)$ & 2 & $33.3 \%(0-71.1 \%)$ \\
\hline
\end{tabular}

Values are presented as $n$ and percentage (95\% confidence interval). MoM, multiple of the expected mean; PDE-5i, phosphodiesterase type 5 inhibitor.

body weight were applied in patients receiving sildenafilmacitentan $(0.96 \pm 0.43 \mathrm{mg} / \mathrm{kg}, 95 \%$ CI $0.82-1.11)$ compared to sildenafil-bosentan $(2.57 \pm 1.54 \mathrm{mg} / \mathrm{kg}, 95 \%$ CI 2.09-3.06; $p<0.001)$ or sildenafil-ambrisentan $(1.98 \pm$ $1.22 \mathrm{mg} / \mathrm{kg}, 95 \%$ CI $1.43-2.53 ; p<0.001)$. However, the individual dose $/ \mathrm{kg}$ was not significantly associated with PDE-5i concentrations (ANOVA, $p=0.19$; Fig. 4). Sensitivity analyses excluding patients with known interacting conditions such as concomitant disease or concomitant medication (imatinib) led to the same results for dis- tribution of medication concentrations and influencing factors.

No significant correlations were found between clinical data and MoM for ERA or PDE-5i.

\section{Effect of Switching ERA on Sildenafil Exposure}

In 20 patients receiving sildenafil-bosentan combination therapy, clinically indicated switching of ERA treatment to macitentan was performed. Reasons for change in medication were unsatisfactory long-term response 


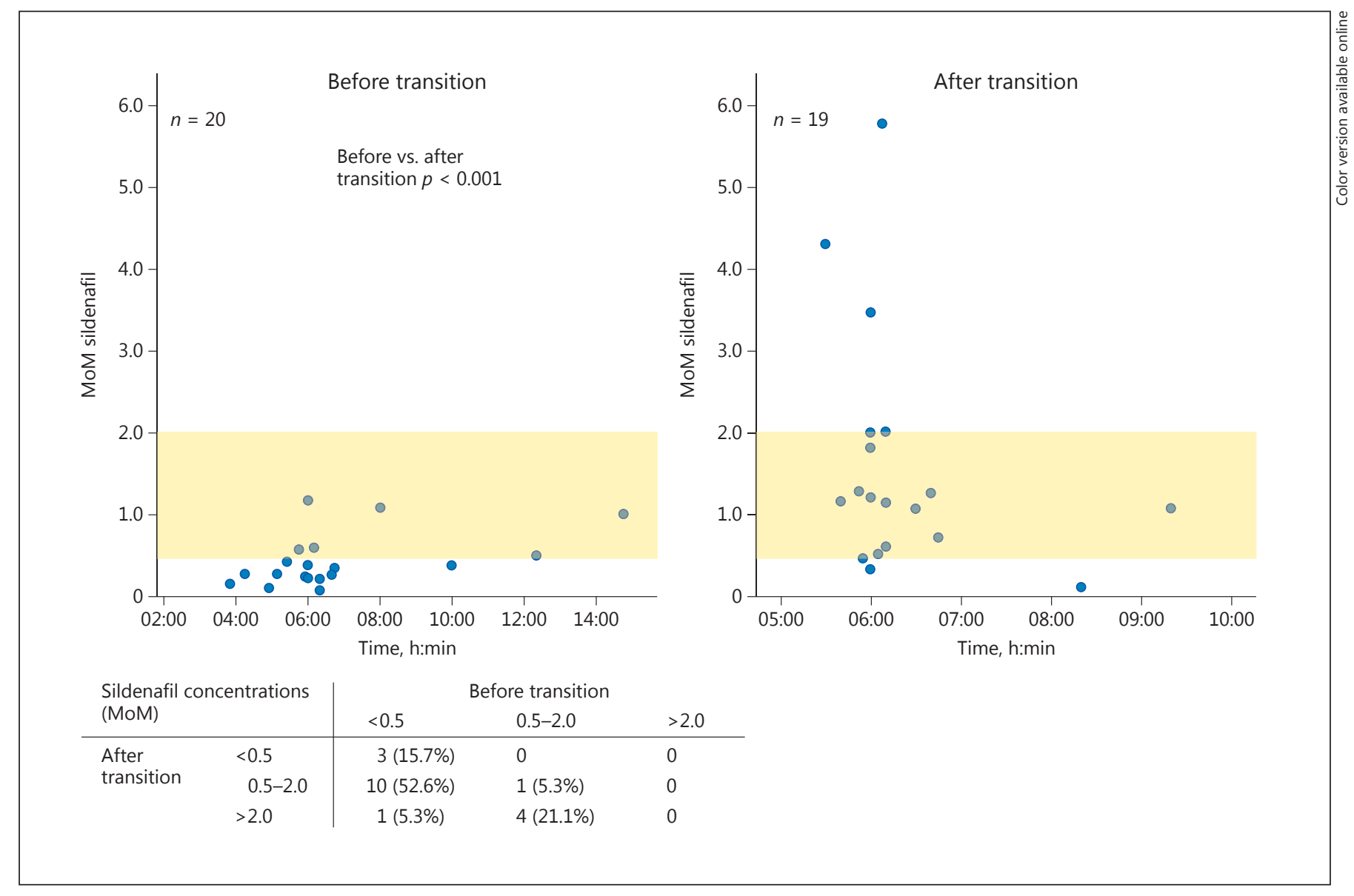

Fig. 5. Change of endothelin receptor antagonist treatment led to a significant increase in sildenafil concentrations $(p<0.001)$. The MoM range between 0.5 (half of expected value) and 2.0 (twice the expected value) is indicated as a shaded area.

$(n=10)$, subjective dissatisfaction with exercise capacity (treatment optimization; $n=4)$, clinical worsening $(n=$ $5)$, and adverse gastrointestinal side effects $(n=1)$. Medication switching was performed independent from drug plasma concentrations. At the time of switching, patients and clinicians did not know the results of medication concentrations.

After switching to macitentan (median, 3 months; range, 1-12 months), sildenafil concentrations showed a significant increase after transition compared to baseline values during bosentan treatment (MoM $0.42 \pm 0.32$, 95\% CI 0.28-0.56, vs. MoM 1.59 $\pm 1.46,95 \%$ CI 0.94-2.25; $p<$ 0.001 ; Fig. 5). Before switching, 14 patients (74\%) had sildenafil concentrations $<0.5 \mathrm{MoM}$ as opposed to only 3 patients (16\%) thereafter. Patients who changed treatment remained clinically stable and showed a significant decrease in NT-proBNP $(2,204 \pm 3,823 \mathrm{ng} / \mathrm{mL}, 95 \%$ CI
$529-3,880$, vs. $1,181 \pm 2,899 \mathrm{ng} / \mathrm{mL}, 95 \%$ CI $-197-2,560$, $p=0.003$; Table 3$)$.

\section{Discussion}

This is the first study investigating the frequency and magnitude of drug-drug interactions and influencing factors in a large group of PAH patients on long-term combination treatment with PDE-5i and ERA. The most crucial substantial interaction was found in the combination of sildenafil-bosentan, with significantly lower concentrations of sildenafil and higher concentrations of bosen$\tan$. The study showed that $>70 \%$ of our PAH patients receiving sildenafil-bosentan combination treatment for more than 1 month revealed insufficient sildenafil plasma concentrations, which were most likely associated with a 
Table 3. Clinical data before and after medication switch from bosentan to macitentan

\begin{tabular}{|c|c|c|c|}
\hline Characteristic & Baseline & Follow-up & $p$ value \\
\hline Sample size & 20 & 19 & \\
\hline \multicolumn{4}{|l|}{ Clinical data } \\
\hline \multicolumn{4}{|l|}{ WHO functional class } \\
\hline II & $6(33.3 \%)$ & $4(26.7 \%)$ & ns \\
\hline III & $12(66.7 \%)$ & $11(73.3 \%)$ & \\
\hline Oxygen saturation at rest, \% & $92.2 \pm 4$ & $93 \pm 4$ & ns \\
\hline NT-proBNP, ng/mL & $2,204 \pm 3,823$ & $1,181 \pm 2,899$ & 0.003 \\
\hline \multicolumn{4}{|l|}{ Echocardiography } \\
\hline Systolic pulmonary arterial pressure, $\mathrm{mm} \mathrm{Hg}$ & $61 \pm 21$ & $60 \pm 20$ & ns \\
\hline Tricuspid annular plane systolic excursion, $\mathrm{mm} \mathrm{Hg}$ & $21.9 \pm 5.4$ & $21.5 \pm 5.9$ & ns \\
\hline \multicolumn{4}{|l|}{ Right ventricular function } \\
\hline Good & $3(16.7 \%)$ & $2(11.1 \%)$ & \\
\hline Slightly impaired & $1(5.6 \%)$ & $1(5.6 \%)$ & \\
\hline Moderately impaired & $7(38.9 \%)$ & $10(55.6 \%)$ & ns \\
\hline Severely impaired & $7(38.9 \%)$ & $5(27.8 \%)$ & \\
\hline \multicolumn{4}{|l|}{ Drug concentration } \\
\hline Sildenafil level, MoM & $0.42 \pm 0.32$ & $1.59 \pm 1.46$ & $<0.001$ \\
\hline ERA level, MoM & $3.75 \pm 3.62$ & not assessable & \\
\hline \multicolumn{4}{|c|}{$\begin{array}{l}\text { Values are presented as mean } \pm \text { standard deviation or } n(\%) \text {. ERA, endothelin receptor antagonist; MoM, } \\
\text { multiple of the expected mean; ns, not significant; NT-proBNP, N-terminal prohormone of brain natriuretic } \\
\text { peptide. }\end{array}$} \\
\hline
\end{tabular}

reduced clinical effect. After transition of bosentan to macitentan, sildenafil concentrations significantly increased into the targeted range or above in $>70 \%$ of the patients. This increase of sildenafil plasma concentrations was associated with a significant $46 \%$ decrease in NT-proBNP levels during follow-up. However, also in the combinations of other ERA and PDE-5i, low PDE-5i concentrations occurred in 1 patient each for tadalafil-macitentan and tadalafil-bosentan (14-18\%), but not in the combination treatment of tadalafil-ambrisentan. Thus, drug-drug interactions may significantly influence plasma concentrations and may be clinically relevant, especially in case of an insufficient clinical response to therapy.

\section{Interaction between PDE-5i and ERA}

Our findings are in line with previous studies in healthy volunteers [5] or PAH patients [7, 8] reporting a significant interaction between sildenafil and bosentan. Sildenafil plasma concentrations decreased by $44 \%$ when coadministered with bosentan and increased in the presence of imatinib [8], a potent inhibitor of CYP3A [34].

The duration of combination treatment seems to play an important role for drug interactions because pretreated patients had more pronounced interactions than pa- tients with newly initiated targeted combination treatment $[5,35]$. Furthermore, it has been shown that higher dosages of bosentan are more likely to cause abnormal hepatic function [36]. Thus, the side effects of bosentan may even be more pronounced over time, with increasing interaction when coadministered with sildenafil.

\section{Clinical Impact of Plasma Concentrations of Targeted ERA/PDE-5i}

Drug interactions can have a clinical impact if the victim drug has a narrow therapeutic index and its plasma concentrations reach low $(<0.5 \mathrm{MoM})$ or very high concentrations ( $>2 \mathrm{MoM})$. However, there are no largerscaled studies yet systematically assessing the clinical impact of different plasma concentrations. Hatano et al. [35] showed in 8 patients with PAH that sildenafil as add-on treatment to bosentan acutely lowers sildenafil concentrations in the blood and results in a less pronounced effect on pulmonary vascular resistance, but a comparable effect on cardiac index compared to monotherapy. In contrast, in our cohort on chronic treatment, changes in clinical parameters before and after switching were not statistically significant, except for a significant decrease in NT-proBNP. 
Our study was not designed and powered to detect clinical effects after a change of ERA treatment. Even though we did not find strong correlations with clinical data, the significant increase in sildenafil concentrations after switching of ERA from bosentan to macitentan is desirable. The results indicate that transition of an ERA might be an option in patients with unsatisfactory longterm clinical response and an alternative to treatment escalation by initiation of a new class of PAH-targeted drugs. In case series, change of treatment due to side effects or unsatisfactory clinical response from bosentan to ambrisentan [37] and sildenafil to tadalafil [38] seemed to be beneficial. In a case report with 7 subjects, sildenafilambrisentan treatment showed higher medication concentrations for sildenafil than combination of sildenafilbosentan and was associated with a higher 6 -min walking distance [39].

In our cohort, combination treatment with sildenafilambrisentan also showed plasma concentrations within the target range. Therefore, we expect that a switch to ambrisentan instead of macitentan might also be beneficial and would lead to reduction of unfavorable medication interactions. In addition, it should be mentioned that also a transition from sildenafil to tadalafil might be an option considered in case of low clinical response, in particular since the AMBITION study [40] published in 2015 showed advantageous effects on clinical outcome for combination of ambrisentan and tadalafil. Further investigations are needed to detect the effect of PDE-5i change on clinical parameters.

It is not known whether higher plasma concentrations lead to more side effects or are linked to better efficacy. Higher dosages have however shown a more pronounced improvement of hemodynamics in sildenafil [41] and a higher impact on exercise capacity and clinical worsening for tadalafil [3]. As treatment with bosentan has to be thoroughly monitored due to potential liver injuries and showed dose-dependent increases in serum aminotransferase abnormalities [36], drug interactions increasing bosentan concentrations have to be carefully checked [33]. Interestingly, we could not detect elevated transaminase levels in combination with increased bosentan concentrations >2.0 MoM. However, individual dosages should be adjusted according to clinical data and reported side effects of the patient. Further studies are needed to address the relevance of high plasma concentrations of both ERA and PDE-5i.

In patients with CTD, significantly lower drug concentrations were detected throughout the different combination therapies. This finding might be a sign of malabsorp-

Plasma Drug Concentrations in Patients with Pulmonary Arterial Hypertension tion, which is a common issue in patients with CTD [42]. Further studies are needed to assess the frequency and clinical impact of lower medication concentrations in CTD.

\section{Pharmacokinetic Data}

Since bosentan is not only metabolized via CYP3A4 and CYP2C9 [9], but also induces CYP3A4 itself, bosen$\tan$ is prone to cause pharmacokinetic interactions with other CYP substrates $[6,11,29,43]$. Since the metabolism of sildenafil and tadalafil predominantly depends on CYP3A4 activity $[12,13,44]$, reduced plasma concentrations were observed when coadministered with bosentan but not with ambrisentan, which lacks CYP-inducing effects [45]. In a study investigating the pharmacokinetics of sildenafil-bosentan in healthy subjects, the pharmacokinetic data showed lower concentrations for sildenafil after 16 days compared to 6 or 10 days of combination treatment [5]. At the same time, sildenafil increases bosentan concentrations, leading to an even more pronounced interaction between the 2 agents. This counterreaction is only known for the combination of sildenafil and bosentan and may also be one reason for higher sildenafil levels in combination with other ERA.

The higher sildenafil doses $/ \mathrm{kg}$ that were found in patients receiving sildenafil-bosentan in our study cohort may have developed due to a decreasing clinical response after several months, when the interaction between the 2 agents was supposedly more pronounced, leading to a clinically indicated increase in sildenafil dosage. In comparison to sildenafil-macitentan, PDE-5i dosages in sildenafil-ambrisentan were also higher, which might also indicate an adaptation of sildenafil over time due to a subjective feeling of decreasing clinical response. As we did not investigate the development of plasma concentrations over time, we may however not draw conclusions about the causality of higher dosages in this treatment group.

In our study, elimination organ dysfunction did not lead to a significant difference in medication concentrations. This may however be influenced by the small sample size of patients with renal or hepatic insufficiency. Larger studies are needed to assess whether elimination organ dysfunction has an impact on medication concentrations in clinical practice.

The finding that administration of imatinib was associated with significantly higher ERA plasma concentrations raises the question whether the clinical impact of imatinib relies on its own intrinsic effect or is based on increased ERA concentrations. This question has also been addressed by Renard et al. [8], who investigated the

Respiration 2017;94:26-37

DOI: 10.1159/000470916 
pharmacokinetic interactions between bosentan, sildenafil, and imatinib, and those findings were in line with ours. They reported that clinical effects did not depend on the level of ERA concentrations between the imatinib group and the placebo group, and concluded that the effect of imatinib could not be caused by only higher ERA concentrations, but was rather due to imatinib itself. In our study cohort, we did not investigate patients on imatinib monotherapy, and therefore a final conclusion cannot be drawn.

\section{Limitations}

The calculation of MoM is a helpful tool supporting the interpretation of individual drug plasma concentrations. However, there are currently no established therapeutic plasma concentration ranges, and data are mainly based on studies including healthy subjects on monotherapy. Based on the results of the SUPER trial [41], dose and thus presumably also concentration changes critically determine the clinical response. We cannot exclude that values $<0.5$ may also have a positive treatment effect, though a medication plasma concentration of less than half of the expected value is certainly not desirable.

Except for the values before and after transition of a small subgroup, we did not investigate the development of drug concentrations over time.

\section{Conclusion}

This is the first study investigating the frequency and magnitude of medication interactions and influencing factors in various combinations of PAH-targeted treatment in clinical practice in a larger cohort. The combination of sildenafil and bosentan showed the strongest interaction with low sildenafil and high bosentan concen- trations. After change of the ERA treatment, sildenafil concentrations showed a significant increase. If clinically indicated, change of ERA treatment might therefore be considered instead of initiation of a triple combination therapy. Further studies are needed to investigate the impact of low drug plasma concentrations, the effect of plasma concentration changes over time, and the causality of influencing factors.

\section{Acknowledgment}

This study was supported by research grants from Actelion Pharmaceuticals Germany GmbH and GlaxoSmithKline.

\section{Financial Disclosure and Conflicts of Interest}

N. Benjamin received speaker honoraria from Actelion and Bayer. E. Grünig received speaker honoraria and consultancy fees from Actelion, Bayer, GSK, United Therapeutics, and Gilead. W.E. Haefeli received speaker honoraria or consultancy fees from Actelion, Aspen, AstraZeneca, Bayer, Berlin Chemie, BMS, Boehringer Ingelheim, Daiichi-Sankyo, Grünenthal, GSK, and Novartis. H. Klose received honoraria and consultancy fees from Actelion, GSK, Pfizer, Bayer, MSD, United Therapeutics, and OMT. S. Harutyunova, B. Egenlauf, J. Ohnesorge, C. Fischer, Y. Enderle, A. Huppertz, and J. Burhenne report no conflict of interest.

\section{Author Contributions}

Conception and design of the study: E. Grünig, W.E. Haefeli. Data acquisition: E. Grünig, J. Ohnesorge, J. Burhenne, Y. Enderle, B. Egenlauf, S. Harutyunova, A. Huppertz, H. Klose. Analysis and interpretation of data: N. Benjamin, C. Fischer, E. Grünig, J. Ohnesorge, B. Egenlauf, S. Harutyunova, J. Burhenne, A. Huppertz, W.E. Haefeli. Drafting of the article: E. Grünig, J. Ohnesorge, N. Benjamin, C. Fischer. Critical revision of the article: all authors. All authors read and approved the final manuscript.

\section{References}

1 Galiè N, Humbert M, Vachiery JL, Gibbs S, Lang I, Torbicki A, et al: 2015 ESC/ERS guidelines for the diagnosis and treatment of pulmonary hypertension. Eur Heart J 2016;37: $67-119$.

2 McLaughlin V, Channick RN, Ghofrani HA, Lemarié JC, Naeije R, Packer M, et al: Bosen$\tan$ added to sildenafil therapy in patients with pulmonary arterial hypertension. Eur Respir J 2015;46:405-413.

3 Galiè N, Brundage BH, Ghofrani HA, Oudiz RJ, Simonneau G, Safdar Z, et al: Tadalafil therapy for pulmonary arterial hypertension. Circulation 2009;119:2894-2903.
4 Hoeper MM, Huscher D, Ghofrani HA, Delcroix M, Distler O, Schweiger C, et al: Elderly patients diagnosed with idiopathic pulmonary arterial hypertension: results from the COMPERA registry. Int J Cardiol 2013;168:871-880.

5 Burgess G, Hoogkamer H, Collings L, Dingemanse J: Mutual pharmacokinetic interactions between steady-state bosentan and sildenafil. Eur J Clin Pharmacol 2007;64:43-50.

6 Wrishko RE, Dingemanse J, Yu A, Darstein C, Phillips DL, Mitchell MI: Pharmacokinetic interaction between tadalafil and bosentan in healthy male subjects. J Clin Pharmacol 2008; 48:610-618.
7 Paul GA, Gibbs JSR, Boobis AR, Abbas A, Wilkins MR: Bosentan decreases the plasma concentration of sildenafil when coprescribed in pulmonary hypertension. Br J Clin Pharmacol 2005;60:107-112.

8 Renard D, Bouillon T, Zhou P, Flesch G, Quinn D: Pharmacokinetic interactions among imatinib, bosentan and sildenafil, and their clinical implications in severe pulmonary arterial hypertension. Br J Clin Pharmacol 2015;80:75-85. 
9 Treiber A, Schneiter R, Häusler S, Stieger B: Bosentan is a substrate of human OATP1B1 and OATP1B3: inhibition of hepatic uptake as the common mechanism of its interactions with cyclosporin A, rifampicin, and sildenafil. Drug Metab Dispos 2007;35:1400-1407.

10 Weiss J, Theile D, Spalwisz A, Burhenne J, Riedel KD, Haefeli WE: Influence of sildenafil and tadalafil on the enzyme- and transporterinducing effects of bosentan and ambrisentan in LS180 cells. Biochem Pharmacol 2012;85: 265-273.

11 Srinivas NR: Clinical drug-drug interactions of bosentan, a potent endothelial receptor antagonist, with various drugs: physiological role of enzymes and transporters. Gen Physiol Biophys 2016;35:243-258.

12 Takahiro R, Nakamura S, Kohno H, Yoshimura N, Nakamura T, Ozawa S, et al: Contribution of CYP3A isoforms to dealkylation of PDE5 inhibitors: a comparison between sildenafil $\mathrm{N}$-demethylation and tadalafil demethylenation. Biol Pharm Bull 2015;38:5865.

13 Hyland R, Roe EGH, Jones BC, Smith DA: Identification of the cytochrome $\mathrm{P} 450$ enzymes involved in the $\mathrm{N}$-demethylation of sildenafil. Br J Clin Pharmacol 2001;51:239-248.

14 Spence R, Mandagere A, Dufton C, Venitz J: Pharmacokinetics and safety of ambrisentan in combination with sildenafil in healthy volunteers. J Clin Pharmacol 2008;48:14511459.

15 Spence R, Mandagere A, Harrison B, Dufton C, Boinpally R: No clinically relevant pharmacokinetic and safety interactions of ambrisen$\tan$ in combination with tadalafil in healthy volunteers. J Pharm Sci 2009;98:4962-4974.

16 Sidharta PN, Giersbergen PLM, Wolzt M, Dingemanse J: Investigation of mutual pharmacokinetic interactions between macitentan, a novel endothelin receptor antagonist, and sildenafil in healthy subjects. Br J Clin Pharmacol 2014;78:1035-1042.

17 Liesenfeld KH, Lehr T, Dansirikul C, Reilly PA, Connolly SJ, Ezekowitz MD, et al: Population pharmacokinetic analysis of the oral thrombin inhibitor dabigatran etexilate in patients with non-valvular atrial fibrillation from the RE-LY trial. J Thromb Haemost 2011;9:2168-2175.

18 Hoeper MM, Barst RJ, Bourge RC, Feldman J, Frost AE, Galiè N, et al: Imatinib mesylate as add-on therapy for pulmonary arterial hypertension: results of the randomized IMPRES study. Circulation 2013;127:1128-1138.

19 Speich R, Ulrich S, Domenighetti G, Huber LC, Fischler M, Treder U, et al: Efficacy and safety of long-term imatinib therapy for pulmonary arterial hypertension. Respiration 2015;89:515-524.

20 Grünig E, Ehlken N, Ghofrani A, Staehler G, Meyer FJ, Juenger J, et al: Effect of exercise and respiratory training on clinical progression and survival in patients with severe chronic pulmonary hypertension. Respiration 2011;81:394-401.
21 Grünig E, Biskupek J, D’Andrea A, Ehlken N, Egenlauf B, Weidenhammer J, et al: Reference ranges for and determinants of right ventricular area in healthy adults by two-dimensional echocardiography. Respiration 2015;89:284293.

22 Enderle Y, Meid AD, Friedrich J, Grünig E, Wilkens H, Haefeli WE, et al: Dried blood spot technique for the monitoring of ambrisentan, bosentan, sildenafil, and tadalafil in patients with pulmonary arterial hypertension. Anal Chem 2015;87:12112-12120.

23 Martin P, Haefeli WE, Martin-Facklam M: A drug database model as a central element for computer-supported dose adjustment within a CPOE system. J Am Med Inform Assoc 2004;11:427-432.

24 Satoh T, Saji T, Watanabe H, Ogawa S, Takehara $\mathrm{K}$, Tanabe N, et al: A phase III, multicenter, collaborative, open-label clinical trial of sildenafil in Japanese patients with pulmonary arterial hypertension. Circ J 2011;75: 677-682.

25 Vachiery JL, Huez S, Gillies H, Layton G, Hayashi N, Gao X, et al: Safety, tolerability and pharmacokinetics of an intravenous bolus of sildenafil in patients with pulmonary arterial hypertension. Br J Clin Pharmacol 2011;71:289-292.

26 Forgue ST, Patterson BE, Bedding AW, Payne CD, Phillips DL, Wrishko RE, et al: Tadalafil pharmacokinetics in healthy subjects. Br J Clin Pharmacol 2006;61:280-288.

27 van Giersbergen PLM, Halabi A, Dingemanse J: Single- and multiple-dose pharmacokinetics of bosentan and its interaction with ketoconazole. Br J Clin Pharmacol 2002;53:589595.

28 Taguchi M, Ichida F, Hirono K, Miyawaki T, Yoshimura N, Nakamura T, et al: Pharmacokinetics of bosentan in routinely treated Japanese pediatric patients with pulmonary arterial hypertension. Drug Metab Pharmacokinet 2011;26:280-287.

29 Markert C, Schweizer Y, Hellwig R, Wirsching T, Riedel K, Burhenne J, et al: Clarithromycin substantially increases steady-state bosentan exposure in healthy volunteers. $\mathrm{Br} \mathrm{J}$ Clin Pharmacol 2014;77:141-148.

30 Markert C, Hellwig R, Burhenne J, Hoffmann MM, Weiss J, Mikus G, et al: Interaction of ambrisentan with clarithromycin and its modulation by polymorphic SLCO1B1. Eur J Clin Pharmacol 2013;69:1785-1793.

31 Dingemanse DJ, van Giersbergen PLM: Clinical pharmacology of bosentan, a dual endothelin receptor antagonist. Clin Pharmacokinet 2012;43:1089-1115.

32 Nichols DJ, Muirhead GJ, Harness JA: Pharmacokinetics of sildenafil after single oral doses in healthy male subjects: absolute bioavailability, food effects and dose proportionality. Br J Clin Pharmacol 2002;53(suppl 1): $5 \mathrm{~S}-12 \mathrm{~S}$.
33 Venitz J, Zack J, Gillies H, Allard M, Regnault J, Dufton C: Clinical pharmacokinetics and drug-drug interactions of endothelin receptor antagonists in pulmonary arterial hypertension. J Clin Pharmacol 2012;52:1784-1805.

34 Filppula AM, Laitila J, Neuvonen PJ, Backman JT: Potent mechanism-based inhibition of CYP3A4 by imatinib explains its liability to interact with CYP3A4 substrates. Br J Pharmacol 2012;165:2787-2798.

35 Hatano M, Yao A, Kinugawa K, Hirata Y, Nagai R: Acute effect of sildenafil is maintained in pulmonary arterial hypertension patients chronically treated with bosentan. Int Heart J 2011;52:233-239.

36 Rubin LJ, Badesch DB, Barst RJ, Galiè N, Black CM, Keogh A, et al: Bosentan therapy for pulmonary arterial hypertension. $\mathrm{N}$ Engl J Med 2002;346:896-903.

37 McGoon MD, Frost AE, Oudiz RJ, Badesch DB, Galiè N, Olschewski H, et al: Ambrisentan therapy in patients with pulmonary arterial hypertension who discontinued bosentan or sitaxsentan due to liver function test abnormalities. Chest 2009;135:122-129.

38 Lichtblau M, Harzheim D, Ehlken N, Marra A, Pinado FP, Grünig E, et al: Safety and longterm efficacy of transition from sildenafil to tadalafil due to side effects in patients with pulmonary arterial hypertension. Lung 2015; 193:105-112.

39 Hakamata A, Odagiri K, Miyakawa S, Irisawa $\mathrm{H}$, Takeuchi K, Inui N, et al: Pharmacokinetic and pharmacodynamic comparison of sildenafil-bosentan and sildenafil-ambrisentan combination therapies for pulmonary hypertension. Clin Transl Sci 2016;9:29-35.

40 Galiè N, Barberà JA, Frost AE, Ghofrani HA, Hoeper MM, McLaughlin VV, et al: Initial use of ambrisentan plus tadalafil in pulmonary arterial hypertension. N Engl J Med 2015;373: 834-844.

41 Galiè N, Ghofrani HA, Torbicki A, Barst RJ, Rubin LJ, Badesch D, et al: Sildenafil citrate therapy for pulmonary arterial hypertension. N Engl J Med 2005;353:2148-2157.

42 Savarino E, Furnari M, de Bortoli N, Martinucci I, Bodini G, Ghio M, et al: Gastrointestinal involvement in systemic sclerosis. Presse Med 2014;43:e279-e291.

43 Weber C, Schmitt R, Birnboeck H, Hopfgartner G, Eggers H, Meyer J, et al: Multiple-dose pharmacokinetics, safety, and tolerability of bosentan, an endothelin receptor antagonist, in healthy male volunteers. J Clin Pharmacol 1999;39:703-714.

44 Warrington JS, Shader RI, von Moltke LL, Greenblatt DJ: In vitro biotransformation of sildenafil (Viagra): identification of human cytochromes and potential drug interactions. Drug Metab Dispos 2000;28:392-397.

45 Markert C, Kastner IM, Hellwig R, Kalafut P, Schweizer Y, Hoffmann MM, et al: The effect of induction of CYP3A4 by St John's wort on ambrisentan plasma pharmacokinetics in volunteers of known CYP2C19 genotype. Basic Clin Pharmacol Toxicol 2015;116:423-428.
Plasma Drug Concentrations in Patients with Pulmonary Arterial Hypertension
Respiration 2017;94:26-37 DOI: $10.1159 / 000470916$ 